\title{
Fuzzy Analysis of Classifier Handshapes from 3D Sign Language Data
}

\author{
Kabil Jaballah and Mohamed Jemni \\ 5 avenue Taha Houssein, ENSIT \\ LaTICE research Laboratory, University of Tunis \\ Kabil.jaballah@utic.rnu.tn, Mohamed.jemni@fst.rnu.tn
}

\begin{abstract}
In this paper, we present a novel technique to track and recognize both classic handshapes and descriptive classifier handshapes inside 3D sign language sequences. Our approach is able to evaluate the intensity of CL-C, CL-L and the CL-G classifiers which are used to specify sizes or amounts of objects. Our method combines Minkowski similarity measures to match the shape of the hand and a fuzzy inference system (FIS) to quantify the classifier's intensity. We implemented and tested our framework on a set of 3D sign language data. The membership functions as well as the rules of the designed FIS were optimized by 12 participants. The system generates evaluations which are very close to human perception of the iconic information conveyed by the classifier handshapes. The correlation of results generated by our system with those awarded by 12 participants is about 0.936 which can be considered as satisfactory.
\end{abstract}

Keywords: 3D sign language, recognition, virtual reality, H-anim, Handshapes, Classifiers, Fuzzy Inference system.

\section{Introduction}

Thanks to 3D signing avatars, users can create 3D signed scenes using customized virtual agents also called "signing avatars". Some of the signing avatars systems are considered as automatic translation machines [1][2][3] able to interpret written texts into ASL (American Sign Language) or other signed languages. Other systems, like $\mathrm{H}$-animator [4], are designed to help people ${ }^{1}$ to create manually their own $3 \mathrm{D}$ signed scenes and share them. These virtual characters have also been used in Virtual Learning Environments (VLE) for deaf children and showed their advantage regarding classic videos of real signers. This is what has been reported in MathSigner [5]. The consequent advances in 3D graphics and the advantages of 3D sign language generation conducted to a huge number of 3D signed contents which are uploaded and exchanged constantly [6]. Thus, the analysis and recognition of these contents constitutes a real research axis aiming to index $3 \mathrm{D}$ sign language and to implement a machine translation to convert $3 \mathrm{D}$ signs into written texts.

\footnotetext{
${ }^{1}$ Deaf individual, their parents, their teacher and all people whom are involving in Sign Language generation.
} 
Automatic sign language recognition is relevant regarding the annotation of signed contents [7] where experts and linguists would be able to efficiently index and build new understandings of the structure of sign language. Parametric recognition approaches aim to recognize separately each sign feature (handshape, orientation, location and movement). In this paper we consider recognizing a specific kind of classifier handshapes where the flexure of specific fingers can alter the classifier's intensity. An example is the CL-G which can be considered as a simple handshape to fingerspell the letter "G" in American Sign Language (ASL) or as a classifier which describes how thin an object is by the possibility to use different intensities. It could be used to show the thinness of a picture frame, a book, a piece of wood, etc. Another classifier to be considered is the CL-C-claw (two handed) which is used for scaling spherical objects (e.g. Ball). We consider also the CL-L-Curved classifier which describes round objects e.g. round table or round mirror. Classifiers describe objects in sign language and bring a level of iconicity which is specific to signed languages [8] [9].

The remainder of the paper is organized as follows. The coming section gives an outline of the most relevant studies and approaches regarding 3D model-based handshapes tracking and recognition dedicated to sign language and robotics. In the section 3, we expose the architecture of the proposed framework. The adopted 3D hand model will be exposed as well as the implemented Fuzzy inference system. In section 4 , we present the 3 classifier handshapes that have been considered in this study. We also describe the data set used for experimentations and bring out the results. Finally, we come up with the conclusion in section 5 .

\section{Related Work}

In the context of sing language, hand recognition and analysis basically focuses on understanding the finger-spelled alphabets and numbers. Many approaches have been proposed to recognize the hand posture. Model-based methods generate 3D model hypothesis of the hand and evaluate them through observations by commonly computing the similarity distances. The complexity of the similarity functions resides on the fact that it handles multidimensional parameters of the model. Moreover, unlike appearance-methods, most of the calculations are executed online. In this context, many researchers proposed to estimate the joints rotations of the fingers and compare them with the projection of the hand model's joints. That is what has been done by Holden and Owen [10] who fetched the hand's joints rotations using colored markers that was placed at the articulations. On the other hand, Dorner [11] estimated the hand pose through a function that uses constraints of possible rotation angles that have been specified in the set of model's templates. Stenger et al [12] used a decision tree to detect the position of the hand. The posture is then classified through a set of model templates and retrieved using the nearest neighbor method. Rezaei et al [13] used a 3D Fourrier descriptor to construct and match a 3D model of the hand. They computed loose point correspondence and 3D motion estimation for the data issued from stereo cameras. Another robust finger tracking system has been proposed in [14] who used combined channels from multiple cameras that track the fingers. The gathered data is then sent to a Bayesian framework which reduces the sum of squared 
differences equation to find the best model which explains observations. Unfortunately, the data set did not include handshape classifiers with different intensities. More recently, the work in [15] proposed a system to recognize signs inside continuous 3D signed scenes. The approach was based on an adaptation of the Longest Common Subsequence algorithm to align a model template with an observation. The similarity distance between extracted 3D key-frames was computed using the Minkowski similarity. Again, the proposed approach didn't consider descriptive classifiers.

\section{Proposed Framework}

In our study, we deal with 3D sign language data that is generated through dedicated signing avatars systems, through 3D devices like data-gloves and through manual methods using $\mathrm{H}$-anim ${ }^{2}$ compliant software. The first step consists on extracting relevant 3D-keyframes from the continuous sign language data (see fig1). These keyframes contain motion and joint angles information of the hand. The extracted data is then normalized and sent to the shape estimator to recognize the configuration of the hand. Simultaneously, the observations are analyzed in order to detect the eventual flexure of the finger or other relevant articulations that could modify the descriptive classifier's intensity. Those two estimators are based on a database of predefined models of 3D handshapes and descriptive classifiers. The shape estimator generates a similarity distance between the observed shape $(\mathrm{O})$ and the stored shape $(\mathrm{S})$. Since the hand model we use is composed of 16 joints, each gathered observation generates a vector of angles of 26 Degree of Freedom (DOF). Subsequently, the similarity distance is calculated through the Minkowski Similarity [16] of order K (K is number of DOF regarding each two compared joints) between the joints that don't affect the classifier's intensity.

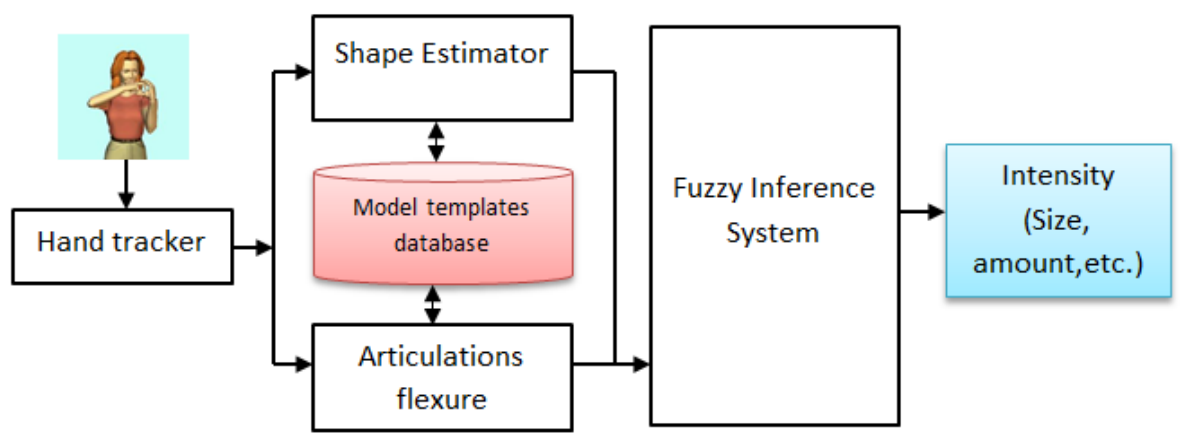

Fig. 1. Proposed Framework architecture

\footnotetext{
${ }^{2}$ http: / /h-anim.org/
} 
The articulations flexure estimator analyzes the joint angles of the articulations that affect the classifier. This module normalizes the measures and sends them to the fuzzy inference system (FIS) once a relevant distance measure has been computed by the shape estimator. The FIS fuzzifies the input values and then applies the designed rules to generate a defuzzified value that expresses the intensity or amount of the descriptive classifier.

\subsection{D Hand Modeling}

The human hand is complex and highly articulated. We have to model a hand able to represent all the anatomically correct features [17] and the possible configurations of the real hand. In this context, several models have been proposed in the literature. Heap and Hogg [18] proposed a deformable point distribution model. Unfortunately, we can't adopt this model since it isn't able to reproduce realistic motions and configurations because it doesn't include a rigid skeleton. A skeleton based model has been introduced in [19] and [20]. This kind of models uses kinematics to represent the joints angles of the hand Degrees of Freedom DOF and to animate the model.

In our work, we use a 3D skeletal model of the hand which is compliant with $\mathrm{H}$-anim ${ }^{3}$. Our model is composed of 3 main parts (see fig3) which are the carpal bones (carpals), the metacarpal bones (metacarpals) and phalangeal bones (phalanges). The resulting hierarchical structure of the model is 26 DOF which allows us to model and store all the handshapes and classifiers for all the signed languages.
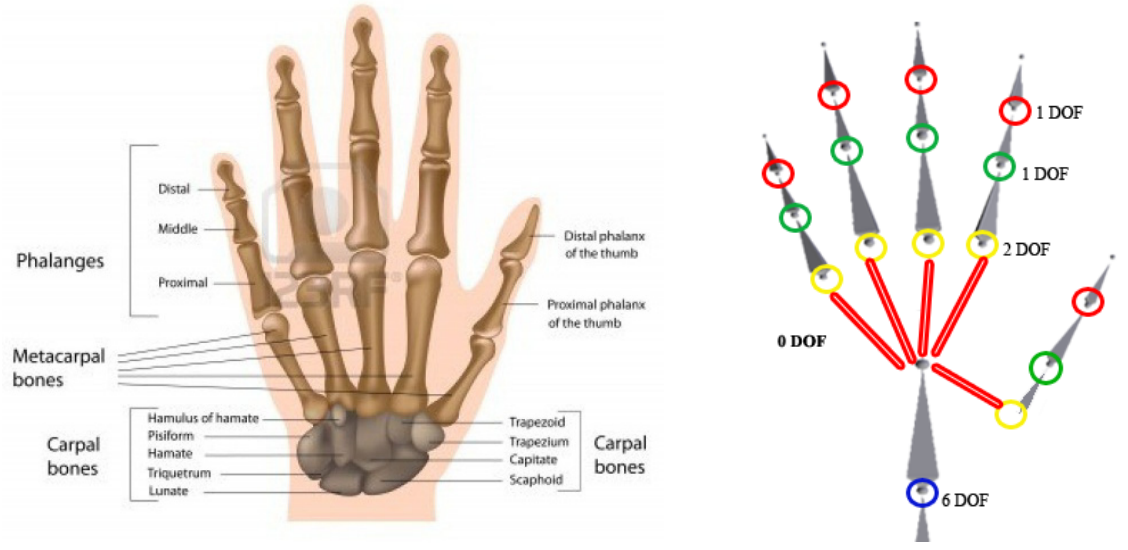

Fig. 2. Hand bones and hierarchy (left), Example of H-anim Hand model with 26 DOF(right)

The palm is designed as a kinematic structure with 6 DOF. 3 DOF to specify the position and 3 DOF for it's the orientation. Regarding the fingers, each one constitutes a 4 DOF kinematic chain. 2 DOF for the metacarpophalangeal joint (MCP) and 2 DOF for the proximal interphalangeal joint (PIP) and the distal interphalangeal joint (DIP).

\footnotetext{
${ }^{3}$ http: / /www.h-anim.org
} 
The benefit of this kinematic model is the fact that we can easily store it a database using XML. The following figure (fig3) shows the adopted structure of our XML file used to stored handshapes and classifiers examples in the database. The main contribution consists on the compliance with the H-anim standards and the fact that the same model supports classic handshapes and classifier handshapes. Moreover, the two handed classifiers could be described by this model as well. This is could be done by specifying the attribute "type" of the "config" tag. If the handshape is a classic one (No descriptive classifier), the attribute "Class" of the node "config" should be left blank and the attributes of the node "classifier" should be left blank as well. As we can see in the following figure, the concerned classifier is the CL-C which could have different intensities depending on the flexure of the "index" and the "thumb".

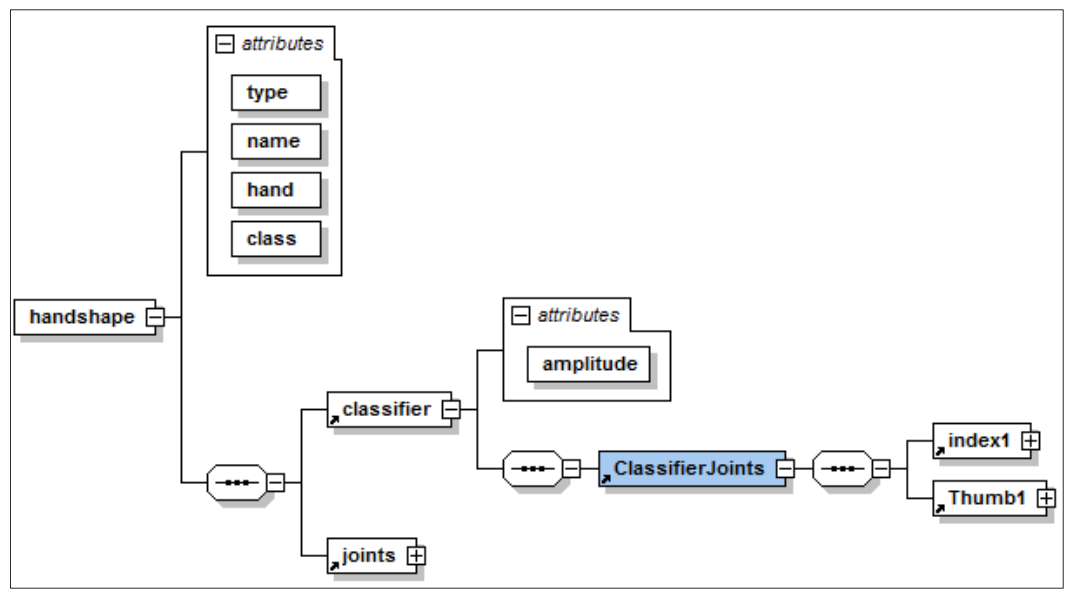

Fig. 3. Example of 3D handshape Classifier structure 'CL-C'

\subsection{Fuzzy Inference System}

- Let each of the joints affecting a classifiers' intensity $\left(j_{n}\right)$ be interpreted as a linguistic variable. The data gathered from this variable is the flexure (openness) of the concerned articulation over an axis of rotation $(x, y, z) .\left(j_{n}\right)$ is then decomposed into the three following terms $T(j 1)=\{$ Close, Neutral,Open $\}$ where each term in $T\left(j_{n}\right)$ is characterized by a fuzzy set in the universe of discourse $U=[\operatorname{MinR}, \operatorname{MaxR}]$. The MinRotationAngle and MaxRotationAngle values are set according to anatomical possibility of a specified joint for performing a given rotation over a specified axis.

- Let a Descriptive Classifier's (DCL) intensity (I) be interpreted as an output linguistic variable resulting from the inference process that includes rule based engine. $(I)$ is decomposed into the following terms $T(I)=\{$ small, normal, big, Very big $\}$ where each term in $T(I)$ is characterized by a fuzzy set in the universe of discourse $U=[0,10]$. For the purpose of defuzzification of (I)into a crisp value expressing the amount/intensity/size, the method of center of gravity is used [21]. 
The membership functions $\mu_{f}(x)$ regarding the designed the variables that we will use are trapezoidal. The design of those functions as well as the rule base have been made by 10 Phd students doing research on virtual reality and 2 sign language experts. They are asked to evaluate and set the degrees of membership for the specified variable based on their visual perception of the $3 \mathrm{D}$ models that have been used in the experimentation. Thus, for each term of a given variable, the limits of membership $\operatorname{kernel}(a, b)$, as well the overlapping parameters $(\alpha, \beta)$ are set by the experts.

The rule base is an important part of the system which links the membership functions of the input premise to the membership function of the output premise. All the combinations of the terms that have been previously designed are implemented and rendered in order to be visualized by the 12 members involved in the evaluation. The rules are then designed according to what has been visualized. The following table1 shows a template of the rules that have been implemented concerning the three descriptive classifiers (fig1) where only two joints angles of rotation are altered while expressing different intensities.

Table 1. Rule base for CL-C-claw, CL-G and CL-L-curved

\begin{tabular}{|l|l|l|}
\hline Joint1 & Joint2 & Classifier intensity \\
\hline O & O & Very Big \\
\hline O & N & Big \\
\hline O & C & Normal \\
\hline N & O & Big \\
\hline N & N & Normal \\
\hline N & C & Small \\
\hline C & N & Small \\
\hline C & C & Small \\
\hline C & O & Normal \\
\hline
\end{tabular}

\section{$4 \quad$ Experimental Results}

The described framework has been implemented using MATLAB ${ }^{4}$ and tested with SIMULINK $^{5}$. We collected a set of 3D sign language data issued from 3 different systems. The common point between all these data is the fact that the modeled avatars rendered are compliant with $\mathrm{H}$-anim. Moreover, all of them generate American Sign Language (ASL) and subsequently include the handshapes and classifiers of the same language. On the one hand, the processed data is composed of scenes grabbed from the internet and/or recorded using Vsigns [22], H-animator [4], WebSign [1] and Pos$\mathrm{er}^{6}$. The scenes generated by each system contain 4 variations of intensity for each of the 3 descriptive classifiers. This gives us 12 examples that will be processed and evaluated by our fuzzy system as well as by the 12 participants. On the other hand, 56

\footnotetext{
${ }^{4}$ www.mathworks. com

5 www.mathworks.com/products/simulink/

${ }^{6}$ http: //poser. smithmicro.com/poser.html
} 
classic handshapes have been modeled (section 3.1) and stored in a database using XML. We obviously used the 3D hand model to create those models and to model the 3 descriptive classifiers as shown in the figure 4 . The created models are used as templates that will be analyzed and matched in the processed continuous $3 \mathrm{D}$ scenes.

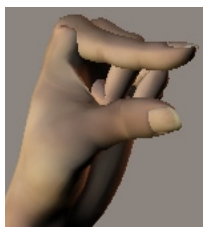

CL-G (S)

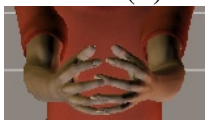

CL-C-claw (S)

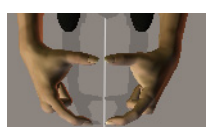

CL-L (S)

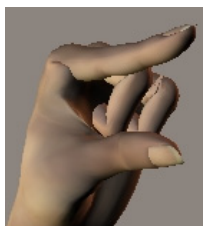

CL-G (N)

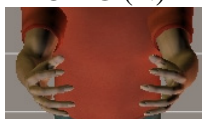

CL-C-claw (N)

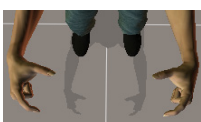

CL-L(N)

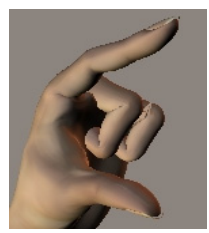

CL-G (B)

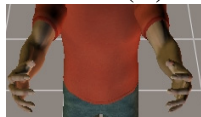

CL-C-claw (B)

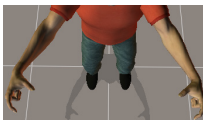

CL-L(B)

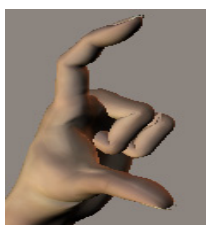

CL-C(VB)

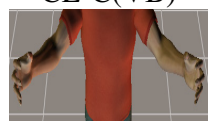

CL-C-

$\operatorname{claw}(\mathrm{VB})$

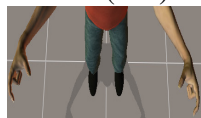

CL-L(B)

Fig. 4. The 3 modeled classifiers with their four intensities Small(S), Normal (N), Big (B) and Very Big (VB)

Table 2. Intensity levels

\begin{tabular}{|c|c|c|c|}
\hline \multirow{2}{*}{\multicolumn{2}{|c|}{ Classifier }} & \multicolumn{2}{|c|}{ Intensity ( 1 to 10$)$} \\
\hline & & Human Evaluation & Fuzzy Evaluation \\
\hline \multirow{4}{*}{ DCL-G } & Small & 2,083 & 1,701 \\
\hline & Normal & 4,307 & 5,843 \\
\hline & Big & 6,35 & 7,90 \\
\hline & Very Big & 7,16 & 8,702 \\
\hline \multirow{4}{*}{ CL-C-Claw } & Small & 2,125 & 1,66 \\
\hline & Normal & 4,66 & 4,002 \\
\hline & Big & 6,5 & 7,00 \\
\hline & Very Big & 7,99 & 8,98 \\
\hline \multirow{4}{*}{ CL-L-curved } & Small & 1,2 & 2,509 \\
\hline & Normal & 4,33 & 3,8 \\
\hline & Big & 6,22 & 5,66 \\
\hline & Very Big & 8,1 & 7,919 \\
\hline
\end{tabular}

To validate our FIS, 4 intensities of each Descriptive classifier were included in the processed scenes. The participants in the evaluation are $10 \mathrm{PhD}$ students doing research in sign language and virtual reality plus 2 experts in sign language translation. 
Thus, the participants had the relevant background which makes us avoid different perceptions among different user groups. The evaluators were shown the collected 3D sign language sequences and asked to focus on the intensity of the classifier if they see one. They awarded scores on a scale from 1 (small) to 10 (very big). They had the possibility to freeze the animation and review it many times if needed. The average score of the 12 evaluators was computed and used as the gold standard. We subsequently computed gold standards variations for all the evaluated scenes.

The correlation between human evaluation and the crisp values generated by our fuzzy system is about 0.939 which is can be considered as satisfactory. This value has been calculated according to the correlation coefficient which is expressed with the following formula:

$$
\operatorname{CorrCoef}(X, Y)=\frac{\sum(x-\bar{x})(y-\bar{y})}{\sqrt{\sum(x-\bar{x})^{2} \sum(y-\bar{y})^{2}}}
$$

Where $\bar{x}$ and $\bar{y}$ are the sample's means of human evaluation array's average and fuzzy evaluation array's average.

As we can notice in the figure 5, the fuzzy evaluation average seems to be slightly higher than human evaluation especially for "Big" and "Very Big" intensities. This can be explained by the fact those classifiers were underscored by the evaluators due to the fact that shapes and sizes of objects are evaluated relatively with the other elements of the scene. In fact, the size of an object is interpreted relative to the objects around it and in the context of the other parameters like perspective, position of the signer etc. the current correlation value has been reached after several optimizations of the membership functions by adjusting the fuzzy sets intervals.

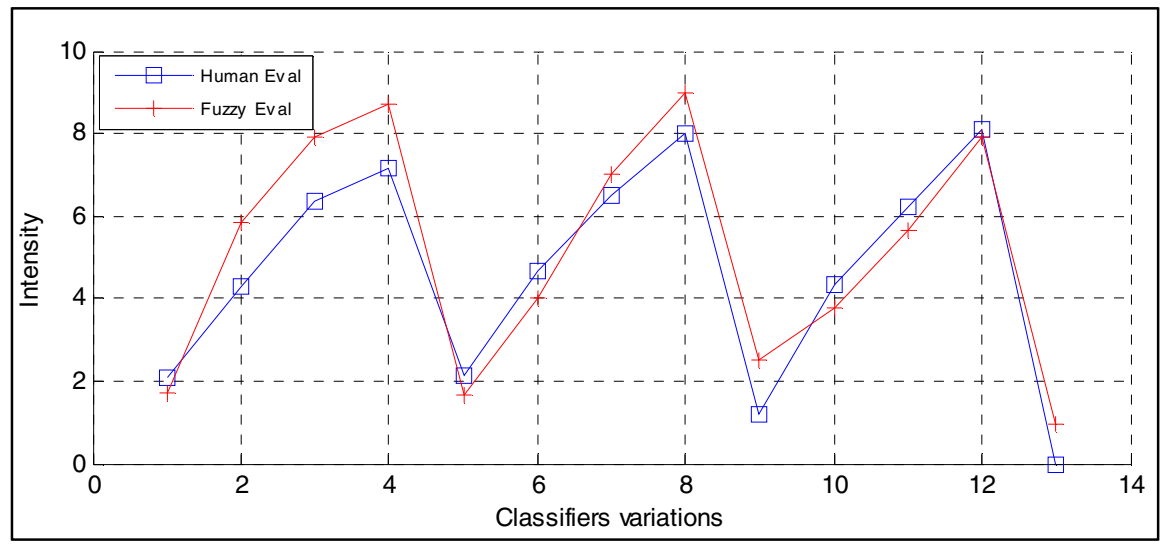

Fig. 5. Human versus fuzzy evaluation of the modeled classifiers intensity

\section{Conclusion and Future Work}

I this paper, we presented a new approach for recognizing and evaluating 3D descriptive classifier handshapes that include different intensities. This study focused on 3 
descriptive classifiers commonly used in sign language. We designed a Fuzzy Inference System that fuzzifies entries extracted from the processed 3D data, based on a set of rules, generates crisp values of an eventual classifier's intensity. The correlation score between human and fuzzy evaluation is 0.939 which is satisfactory and very encouraging. In the future, this value could be enhanced by combining a knowledge base as well as a new strategy for optimizing the fuzzy sets intervals. Unfortunately, at time of writing, there are no enough $3 \mathrm{D}$ sign language data to implement training based technique like neural and bayesian for recognizing classical handshapes. For this reason, we started to collect this kind of scenes in order to construct a 3D sign language corpus in the near feature.

\section{References}

1. Jemni, M., ElGhoul, O.: A System to Make Signs Using Collaborative Approach. In: Miesenberger, K., Klaus, J., Zagler, W.L., Karshmer, A.I. (eds.) ICCHP 2008. LNCS, vol. 5105, pp. 670-677. Springer, Heidelberg (2008)

2. Bangham, J., Cox, S., Elliott, R., Glauert, J., Marshall, I., Rankov, S., Wells, M.: Virtual Signing: Capture, Animation, Storage and Transmission - An Overview of the ViSiCAST Project. In: IEE Seminar on Speech and Language Processing for Disabled and Elderly People, London (2000)

3. Papadogiorgaki, M., Grammalidis, N., Sarris, N., Strintzis, M.G.: Synthesis of Virtual Reality Animations from SWML using MPEG-4 Body Animation Parameters. In: Processing Workshop, Lisbon (2004)

4. Buttussi, F., Chittaro, L., Nadalutti, D.: H-Animator: A Visual Tool for Modeling, Reuse and Sharing of X3D Humanoid Animations. In: International Conference on 3D Web Technology, New York (2006)

5. Adamo-Villani, N., Doublestein, J., Martin, Z.: Sign Language for K-8 mathematics by 3D interactive animation. Journal of Educational Technology Systems 33(3) (2005)

6. Jaballah, K., Jemni, M.: Towards Sign language indexing and Retrieval. In: CSUN, San Diego, CA (2011)

7. Dreuw, P., Ney, H.: Towards automatic sign language annotation for the elan tool. In: Int. Conf. LREC Wkshp.: Representation and Processing of Sign Languages (2008)

8. Cuxac, C.: La langue des signes française, les voies de l'iconicité. Orphys (2000)

9. Frishberg, N.: Arbitrariness and Iconicity: Historical Change in American Sign Language. Language 51(23) (1975)

10. Holden, E., Owens, R.: Visual Sign Language Recognition. In: Workshop Theoretical Foundations of Computer Vision (2000)

11. Dorner, B.: Chasing the Colour Glove: Visual Hand Tracking. Simon Fraser Univ. (1994)

12. Stenger, B.: Template-based hand pose recognition using multiple cues. In: Narayanan, P.J., Nayar, S.K., Shum, H.-Y. (eds.) ACCV 2006. LNCS, vol. 3852, pp. 551-560. Springer, Heidelberg (2006)

13. Rezaei, A., Vafadoost, M., Rezaei, S., Daliri, A.: 3D Pose Estimation via Elliptical Fourier Descriptors for Deformable Hand Representations. In: The 2nd International Conference on Bioinformatics and Biomedical Engineering, ICBBE 2008, 16-18, pp. 1871-1875 (2008) 
14. Jennings, C.: Robust finger tracking with multiple cameras. In: Workshop on Recognition, Analysis, and Tracking of Faces and Gestures in Real-Time Systems, Corfu, Greece (1999)

15. Jaballah K., Jemni M.: Toward Automatic Sign Language Recognition from Web3D Based Scenes. In: International Conference in Computers Helping People with Special Needs, Vienna (2010)

16. Deza, E., Deza, M.: Dictionary of Distance. Elsevier (2006)

17. Albrecht, I., Haber, J., Seidel, H.-P.: Construction and Animation of Anatomically Based Human Hand Models. In: Proceedings of the 2003 ACM SIGGRAPH/Eurographics Symposium on Computer Animation, pp. 98-109 (2003)

18. Heap, T., Hogg, D.: Towards $3 \mathrm{~d}$ hand tracking using a deformable model. In: Proceedings of the Second International Conference on Automatic Face and Gesture Recognition, pp. 140-145 (1996)

19. Bray, M., Koller, M., Schraudolph, N., Gool, L.V.: Stochastic meta-descent for tracking articulated structures. In: Conference on Computer Vision and Pattern Recognition Workshop, CVPRW 2004, p. 7 (2004)

20. Wu, Y., Huang, T.S.: Vision-based gesture recognition. In: Braffort, A., Gibet, S., Teil, D., Gherbi, R., Richardson, J. (eds.) GW 1999. LNCS (LNAI), vol. 1739, pp. 103-115. Springer, Heidelberg (2000)

21. Ross, T.J.: Fuzzy logic with engineering applications. McGraw Hill International (1997)

22. Papadogiorgaki, M., Grammalidis, N., Sarris, N., Strintzis, M.G.: Synthesis of Virtual Reality Animations from SWML using MPEG-4 Body Animation Parameters. In: Proceeding of: Workshop on the Representation and Processing of Sign Languages, 4th International Conference on Language Resources and Evaluation (2004) 